\title{
Determining Association of Mental Wellbeing and Resilience among Pakistani Medical Students \& the Perceived Effect on Their Future Behaviour as a Doctor
}

\author{
${ }^{1}$ Dr. Tahira Amjad, ${ }^{2}$ Abdul Haseeb Nawaz Satti, ${ }^{3}$ Bilal Ahmed, ${ }^{4}$ Ali Hassan, ${ }^{5}$ Bashir Ahmed \& \\ ${ }^{6}$ Haamid Khan \\ ${ }^{1}$ Asst. Prof Community Medicine, Pakistan \\ 2, 3,4,5,6 MBBS Student, Pakistan
}

\begin{abstract}
Background: Mental health conditions are on the rise. In Pakistan, the mental health of university students is suboptimal especially among medical students. This study aims to identify the mental well-being and resilience among Pakistani medical students and to establish a relationship between resilience and mental well-being.

Method: It was a cross-sectional study conducted in FUMC. Simple random sampling was done. Students from 2nd to 5th year MBBS were included in the study via lottery method. A structured self-administered questionnaire was used for data collection. The questionnaire comprised of Warwick Edinburgh Mental Well-Being Scale and Brief Resilience Scale as well as 9 statements that assessed the perception of medical students.
\end{abstract}

Results: The mean WEMWB score was $47.41 \pm 8.87$ and the mean resilience score was 3.09 \pm 0.68 . $24.8 \%$ of medical student fell into low mental well-being category and $40.20 \%$ of medical students fell into low resilience category. The association between mental well-being and resilience came out to be significant. $83.3 \%$ of students believed that mental well-being and resilience will have an effect on their future behaviour as doctors. $80.1 \%$ of students agreed on peer interventions to improve resilience and mental well-being of medical students.

Conclusion: The overall mental well-being and resilience status of medical students was normal. However a significant percentage of medical students scored low on mental wellbeing and resilience scales. This study also defined a significant relationship between mental well-being and resilience. Interventions should be done to improve the future behaviour of medical students as doctors.

Keywords: Mental Health; Recommendations; Students; Perception; Interventions.

\section{Introduction}

In the fast paced and growing global world of today, mental health has become an important concern and therefore a progressive frontier for scientific research. In our current generation with a growing population and a rising level of competition the status of mental wellbeing is 


\section{2nd International Conference on Research in SOCIAL SCIENCES and HUMANITIES}

negatively affected amongst multiple diverse groups across the globe. Day by day increasing amount of effort and resources are being dedicated to this field, to understand the fundamentals of mental health and to find solutions for its complex issues.

The global statistics regarding mental health conditions speak for themselves and they present a very bleak picture of the situation. Around 450 million people currently suffer from such conditions, placing mental disorders among the leading causes of ill-health and disability worldwide. Globally, an estimated 300 million people are affected by depression. [1] The statistical data collected from healthcare professionals regarding their mental health statuses presents an even more desolate interpretation. For example, a study from rural British Columbia reported that $80 \%$ of physicians suffered from moderate to severe Emotional Exhaustion, $61 \%$ suffered from moderate to severe Depersonalization, and $44 \%$ had moderate to low feelings of Personal Accomplishment. [2] There appear to be multiple researches conducted over the world, all of whom come down to the same conclusion; Mental Health is a global issue and it's highly concentrated in healthcare community.

Regional researches also gave supporting evidence that mental health of medical students was being affected. For e.g. according to a Hong Kong research. The prevalence of anxiety and depression at Year 3 (University) was 7.5\% and 9.4\%, respectively. [3] Another research from Adelaide Australia reported that, $47.9 \%$ of medical students were psychologically distressed. [4]

Local Pakistani researches presented identical findings regarding mental health as the international research. A Pakistani research conducted among university students reported that, Results indicated relatively poorer mental health and access to mental health treatments. [5] Another Pakistani research concluded that, mental health of medical students is suboptimal, especially among female students. [6]

Recent studies of the prevalence of anxiety among medical students revealed that between 40 and $79 \%$ of medical students experience high levels of anxiety. This is in comparison to approximately $14 \%$ in the general population of the same age group. In addition, depression among medical students is more prevalent and higher than among students pursuing other graduate degrees. Estimate of the prevalence of depression in medical school range from 2 to $35 \%$. [7]

Statistics like these are highly concern raising. They lead to questions like; why is mental health such a huge problem in this community? What are the factors affecting the mental health of medical students? And what interventions are possible to bring a positive change in the current situation?

Keeping in mind the importance of mental health and international focus this subject has received. We have devised a research in which, first of all we aim to assess the mental wellbeing and resilience of medical students. This will give us separate trends for this group. Secondly using these trends we will compare the mental wellbeing and resilience of different years of MBBS. Thirdly by overlapping the acquired data of both aspects of mental health, we will establish if mental wellbeing has any relationship with resilience. If there is indeed a relationship, then of what type is it? Direct, indirect or reciprocal. Our final aim is to assess the perception of the medical students regarding the effect of their mental wellbeing and resilience at this current stage, on their behavior as doctors in the future. 


\section{2nd International Conference on Research in SOCIAL SCIENCES and HUMANITIES}

\section{Rationale of Study}

The rationale of such a large study is a complex one. It has multiple aspects all intermingled with one another. Levels of poor mental health are generally on the rise, particularly among the young and more so in medical students. It can potentially compromise optimal patient care in future. Medical education should prepare the next generation of doctors for the realities of clinical practice and provide an environment to raise their mental wellbeing and resilience. Through literature search, not enough work on this aspect has been done. Though various studies have been done on mental wellbeing and resilience of medical student's effect on their academics. This research will help in identifying the mental wellbeing and resilience among the medical students and will also help in defining a relationship between resilience and mental wellbeing.

The results will enable recommendations for appropriate interventions at the undergraduate level with the aim to improve mental wellbeing and resilience, and its effect on their future behavior as doctors. This research is intended to be a sort of a screening tool with which we aim to locate students who score low on mental wellbeing or resilience scales. The scores will be communicated to desirous students which will give them an opportunity for further evaluation and management.

\section{Objectives:}

1. To assess the Mental Wellbeing status of medical students.

2. To assess the Resilience status of medical students.

3. To determine the relationship between Mental Wellbeing and Resilience.

4. To identify the perception of medical students regarding the effect of Mental Wellbeing \& Resilience at this current stage on their future behavior as doctors.

5. To inform and guide those individuals with low scores of Mental Wellbeing and/or Resilience for professional help.

\section{Operational Definitions}

1. Mental Wellbeing: "State of well-being in which every individual realizes his or her own potential, can cope with the normal stresses of life, can work productively and fruitfully, and is able to make a contribution to her or his community." [8]

2. Categorization will be based upon the scores achieved by the subject on the WarwickEdinburgh Mental Wellbeing Scale \& then using the NHS direct defined cut points [9] in the WEMWS scores. We will categorize the subjects into three categories: Low, Medium \& High Mental Wellbeing.

\begin{tabular}{|c|c|}
\hline WEMWBS SCORES & MENTAL WELL-BEING CATEGORY \\
\hline $40 \&$ Below & Low \\
\hline 41 to 58 & Medium \\
\hline $59 \&$ Above & High \\
\hline
\end{tabular}




\section{2nd International Conference on Research in SOCIAL SCIENCES and HUMANITIES}

1. Resilience: "Is the process of adapting well in the face of adversity, trauma, tragedy, threats or significant sources of stress. It means "bouncing back" from difficult experiences". [10]

2. Interpretation and categorization of Brief Resilience Scale scores (1 -5) will be based upon cut points provided by the scales own authors: Smith \& Colleagues. [11]

\begin{tabular}{|c|c|}
\hline BRIEF RESILIENCE SCALE SCORES & RESILIENCE CATEGORY \\
\hline $1.00-2.99$ & Low \\
\hline $3.00-4.30$ & Normal \\
\hline $4.31-5.00$ & High \\
\hline
\end{tabular}

\section{Materials and Methods}

Study Design: Cross-Sectional Study

Study Population: Medical students of FUIC

Site and Setting: Foundation University Medical College, Foundation University, Islamabad Campus

Duration of Study: 25th May to 30th September 2019 (16 weeks)

Sampling Technique: Simple Random Sampling was done. A representative sample of students from 2nd, 3rd, 4th \& 5th Year MBBS was included in the study via lottery method, giving each subject equal chance of being included in the study. Probability Proportionate to size of the MBBS classes was calculated \& included in the study.

Sample Size: Sample size of 246 was taken with level of confidence 95\%, margin of error $5 \%$, p-value $\leq 0.05$ and response distribution $50 \%$ by using sample size calculator SSCPS Version 1001 (Raosoft Inc) for finite population of 679 medical students (2nd Yr to 5th Yr) at FUIC.

Inclusion Criteria: Students of MBBS at FUMC, from 2nd Year to 5th Year, Who consented to participate in the study \& were present at the time of data collection.

Exclusion Criteria: Students at FUIC who are not from MBBS department, 1st Year MBBS students and those who have not experienced a single professional exam yet.

Data Collection Tool:A structured self-administered questionnaire with open and closeended questions was used for data collection after taking ethical approval from Ethical Review Committee of Foundation University Islamabad (FUI). Consent of the respondents was taken prior for data collection. Moreover, consent for conveying of mental wellbeing and resilience scores was also taken.

The questionnaire comprised of four sections.

Section A was regarding demographic information of the respondents.

Section B included the Warwick-Edinburgh Mental Wellbeing Scale (WEMWBS), which is a validated and accurate 14 item questionnaire, that assess the respondent's mental wellbeing by assigning them a score in the range of 14 to 70 based on the answers. The permission to use this scale is attained by free registration with the Warwick Medical College Online. 


\section{2nd International Conference on Research in SOCIAL SCIENCES and HUMANITIES}

Section C included the Brief Resilience Scale (BRS), which is a validated and accurate 6 item questionnaire designed by The Ohio State University in order to assess the resilience of a respondent by assigning them a score 1 to 6 based on their answers. This is an open access free questionnaire, to use the questionnaire the authors require to be properly referenced.

Section D included 9 statements that assessed the perception of medical students regarding the effect of Mental Wellbeing \& Resilience on their future behavior as doctors on a 5 point Likert scale from strongly disagree to strongly agree.

\section{Data Collection Procedure:}

The questionnaire was conveniently pretested on 15 students of another medical college for its face validity. Amendments were done and then the final questionnaire was distributed among medical students of FUIC, selected by simple random sampling. They filled the questionnaire by themselves and return them to the researcher and on an average 10 to 15 minutes were taken by each respondent.

\section{Data Analysis:}

Scoring and categorizing of both scales (WEMWBS \& BRS) was done on the questionnaire by the researchers themselves, after they had been returned by the respondents.

All the Data analysis was done on Statistical Package for Social Science Version 21(SPSS version 21). Descriptive statistics was applied to compute frequencies and percentages. Inferential statistical analysis was done to see the relationship between them.

\section{Limitations of Study}

1. Due to Time constraints, we were unable to collect data from the total population of FUIC medical students from 2nd to Final year.

2. Study setting was selected out of convenience.

3. Other Medical colleges (Public and Private) were not included; hence our results can't be generalized except the study setting.

4. Free Brief Resilience scale (BRS) was used which was the 3rd best scale available due to financial constraints.

5. Free 14-point WEMWS scale was used instead of 38 point scale as it was time consuming for the respondents.

6. No clinical examination was done to evaluate the psychiatric status of medical students due to ethical considerations.

\section{Results}

This study was conducted on 246 medical students with a response rate of $100 \%$. Mean age of the medical students was 22 years \pm 1.61 . Females were $171(69.5 \%)$ (Table: 1$)$. The Mean WEMWB Score was $47.41 \pm 8.87 \&$ the Mean Resilience Score was $3.09 \pm 0.68$ (Table: 2). The Number of Respondents wishing to be informed of their scores was 102(41.46\%) (Table: 1). Low Category of Mental Wellbeing was reported for $24.8 \%$ students (Figure: 1). Whereas Low Category of Resilience was reported for $40.2 \%$ students (Figure: 2). The Chi Square test P value for cross table of Mental Wellbeing Category \& Resilience Category was .000 \& the Chi Value was 82.019a (Table:3). 


\section{2nd International Conference on Research in SOCIAL SCIENCES and HUMANITIES}

BUDAPEST, HUNGARY

$10-12$ December 2020

Table - 1: Demographic Characteristics of the Respondents. $n=246$

\begin{tabular}{|c|c|}
\hline VARIABLES & RESULTS \\
\hline Age (Years) & $22 \pm 1.61($ Mean \pm SD $)$ \\
\hline Gender Distribution & $n=246(100 \%)$ \\
\hline a. Male & $75(30.50 \%)$ \\
\hline b. Female & $171(69.50)$ \\
\hline MBBS Year Wise Distribution & $n=246(100 \%)$ \\
\hline a. $2^{\text {nd }}$ Year & $60(24.39 \%)$ \\
\hline b. $3^{\text {rd }}$ Year & $60(24.39 \%)$ \\
\hline c. $4^{\text {th }}$ Year & $63(25.60 \%)$ \\
\hline d. $5^{\text {th }}$ Year & $63(25.60 \%)$ \\
\hline Residence Distribution & $\mathrm{n}=246(100 \%)$ \\
\hline a. Boarders & $72(29.3 \%)$ \\
\hline b. Non-Boarders & $174(70.7 \%)$ \\
\hline Dependent Household Members & $3.72 \pm 1.70$ \\
\hline Total Number of Siblings & $2.88 \pm 1,65$ \\
\hline $\begin{array}{l}\text { Number of Respondents Who Wished to be } \\
\text { Informed of their Scores }\end{array}$ & $102(41.46 \%)$ \\
\hline
\end{tabular}

Table - 2: Mean Mental Wellbeing \& Resilience Scores

\begin{tabular}{|l|l|}
\hline VARIABLE & RESULTS (MEAN \pm SD) \\
\hline Mean WEMWB Score & $47.41 \pm 8.87$ \\
\hline Mean Resilience Score & $3.09 \pm 0.68$ \\
\hline
\end{tabular}




\section{SOCIAL SCIENCES and HUMANITIES}

Figure - 1: Mental Wellbeing Category $n=246$

HIGH: $165-67.1 \%$

\section{Figure -}

$\underline{n=246}$
LOW: $20-8.1 \%$

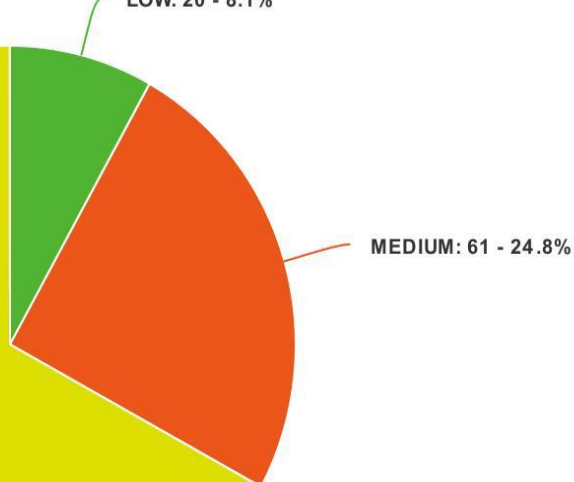

$\underline{\text { Resilience }}$

Category

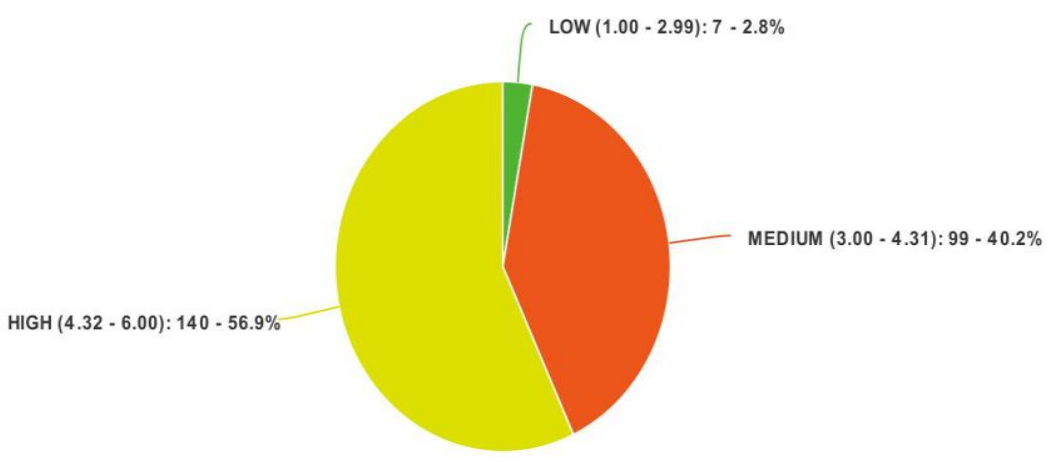

LOW [1.00 - 2.99] MEDIUM (3.00 - 4.31) HIGH [4.32 - 6.00]

\begin{tabular}{|l|l|l|l|l|}
\hline $\begin{array}{l}\text { Chi Square } \\
\text { Test }\end{array}$ & Chi Value & df & P Value & Inference \\
\hline $\begin{array}{l}\text { Mental } \\
\text { Wellbeing } \\
\text { Category \& } \\
\text { Resilience } \\
\text { Category }\end{array}$ & $82.019 \mathrm{a}$ & 4 & .000 & Significant \\
\hline
\end{tabular}

Table - 3: Relationship between Mental Wellbeing \& Resilience Category 


\section{2nd International Conference on Research in SOCIAL SCIENCES and HUMANITIES}

Figure - 3: Relationship between Mental Wellbeing Category \& Resilience Category

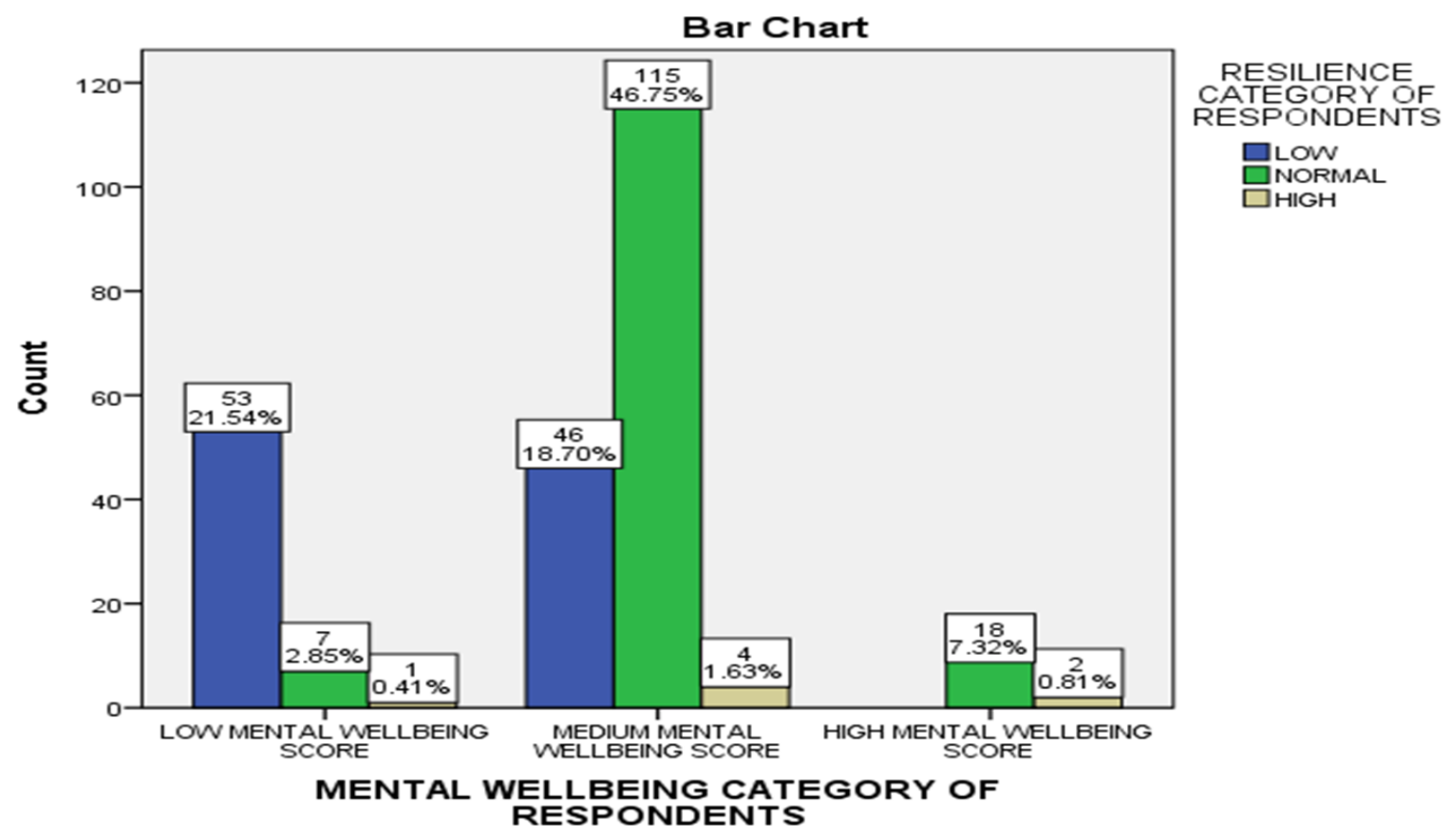

Table - 4: Perception of medical students regarding the effect of Mental Wellbeing \& Resilience on their future behavior as doctors on Likert scale.

\begin{tabular}{|l|l|l|l|}
\hline$\underline{\underline{\text { So }}}$ & STATEMENTS & DISGAREE (\%) & AGREE (\%) \\
\hline 1 & $\begin{array}{l}\text { The mental wellbeing of medical } \\
\text { students has an effect on their future } \\
\text { behaviour as doctors. }\end{array}$ & 16.7 & 83.3 \\
\hline 2 & $\begin{array}{l}\text { The resilience of medical students has } \\
\text { an effect on their future behaviour as } \\
\text { doctors. }\end{array}$ & $\begin{array}{l}\text { A } \\
\text { A physician's mental wellbeing is } \\
\text { directly proportional to the } \\
\text { effectiveness of the organization in } \\
\text { which they practice. }\end{array}$ & 84.5 \\
\hline 4 & $\begin{array}{l}\text { A physician's mental wellbeing is } \\
\text { directly proportional to the quality of } \\
\text { health care they provide to the patient. }\end{array}$ & 16.6 & 83.3 \\
\hline 5 & $\begin{array}{l}\text { Interventions to improve resilience of } \\
\text { medical students at undergraduate } \\
\text { level will have a positive effect on } \\
\text { their future behaviour as doctors. }\end{array}$ & 88.6 \\
\hline 6 & $\begin{array}{l}\text { Interventions to improve mental } \\
\text { wellbeing of medical students at }\end{array}$ & 9.7 & 90.3 \\
\hline
\end{tabular}




\section{2nd International Conference on Research in SOCIAL SCIENCES and HUMANITIES}

\begin{tabular}{|c|c|c|c|}
\hline & $\begin{array}{l}\text { undergraduate level will have a } \\
\text { positive effect on their future } \\
\text { behaviour as doctors. }\end{array}$ & & \\
\hline 7 & $\begin{array}{l}\text { Academic interventions to improve } \\
\text { resilience \& mental wellbeing of } \\
\text { medical students will have a positive } \\
\text { effect on their future behaviour as } \\
\text { doctors. }\end{array}$ & 15 & 85 \\
\hline 8 & $\begin{array}{l}\text { Peer interventions to improve } \\
\text { resilience \& mental wellbeing of } \\
\text { medical students will have a positive } \\
\text { effect on their future behaviour as } \\
\text { doctors. }\end{array}$ & 19.9 & 80.1 \\
\hline 9 & $\begin{array}{l}\text { Institutional interventions to improve } \\
\text { resilience \& mental wellbeing of } \\
\text { medical students will have a positive } \\
\text { effect on their future behaviour as } \\
\text { doctors. }\end{array}$ & 17.5 & 82.5 \\
\hline
\end{tabular}

\section{Discussion}

Our research was conducted to study the perception of medical students on mental wellbeing and resilience in regard to their future as doctors in Pakistan. In our study, we had a sample size of 246 medical students while a similar research conducted in Pakistan in 2019 had a sample size of 1,271 health care professionals and both of the researches had an almost exact result when the mean score of WEMWBS was calculated. [12] Our questionnaire was returned with a $100 \%$ response rate because it took less than 10 minutes to fill out and they were filled out while we were present.

Also in this research they found that males had higher mental well-being scores when compared to females. Once again the results of our research matched with the results of this research on the pattern of WEMWB score \& gender. There are other studies which have also shown that males have more resilience than females. This is most likely multifactorial; one simple factor may be that males show more resilience since society expects that of them from childhood. Other possible factors could be that students face a lot of academic pressure, long study hours and a highly demanding course. It may also be possible that this is the result of questionnaire bias; however that is unlikely since multiple studies show the same result.

Another important result in our study was the significance between resilience and class wise distribution, showing that as medical students pass on to the following class year, their resilience gets a slight bump up. This makes sense, as every year medical students become more comfortable with the college system and the exams. By the time they are in final year, most medical students will have the highest resilience score of their medical college career. Hopefully as they go on to become doctors, their resilience scores will continue to increase. The mean BRS score for our sample was lower than the general population mean calculated by the reference study. In another study as well, medical students were found to have less resilience than the general population. [13] So although medical students have the highest 


\section{2nd International Conference on Research in SOCIAL SCIENCES and HUMANITIES}

resilience score in their final year, the average medical student still have less resilience than the general population. This is quite a surprise because the expectation of medical students is to be exceptionally resilient when faced with stressful situations. After all, these are future medical and surgical doctors in the making and must be able to deal with the life threatening situations when a patient is in their care. Having a clear mind and being able to react quickly in certain scenarios can mean life or death.

The perception of the medical students in our study found that most students felt mental well-being and resilience does have an effect on their future as doctors. The majority believe they can become better doctors if they are mentally well and have high resilience. They also felt more comfortable speaking to colleagues about their issues rather than to the teachers.[13] Universities can use these findings to start programs in which mental wellbeing and resilience can be addressed and techniques can be taught to students on how to increase both of these variables within their lives.

These studies all comment on mental wellbeing and resilience of their subjects. However, they all fail to discuss the relationship between these two. Our research goes a step further than the others and the results show a significant relationship between the variables; Mental Wellbeing Category and Resilience Category of medical students. During the literature search, we didn't come across another study that compared these two variables in such as way.

Due to the limitation of our study in regards to the sample size and restricted location of FUMC, another study should be completed to represent the whole of the Pakistani medical students. In this way, a country wide endeavor can be embarked upon in which medical colleges put an effort into locating students with low mental well-being and resilience so as to help them and create stronger doctors for the future.

\section{Recommendations}

After careful deliberation over the results of our research and the feedback attained from the medical students regarding their mental health situation. We have devised the following recommendations:

\section{Annual Assessment:}

An annual assessment of mental health status should be carried out by the university which includes all of the students.

Previous annual assessments will be used as a comparison to make sure that, the results are improving and no negative trends are seen.

Previous annual assessments may be discarded if they are older than 5 years.

\section{Overall Interventions:}

\section{A. Peer Interventions:}

Colleges can set up student based groups that are overlooked by a professional counselor where students can help each other with some problems they may be facing.

\section{B. Institutional Interventions:}

On campus entertainment/relaxation areas where students can enjoy their breaks.

University remodeling to make it a place pleasing to be in. 


\section{2nd International Conference on Research in SOCIAL SCIENCES and HUMANITIES}

\section{Academic Interventions:}

Making academics less stressful by focusing on learning and understanding rather than memorization.

\section{Individual Interventions:}

Students who have been found to have low mental wellbeing scores must have a professional counselor available to them by the university.

Extra care and attention to be given to these students by faculty e.g. speaking to them in a comfortable way, not picking them out in class, involving their parents if necessary.

\section{Conclusion}

Based upon the results of our research, we drew the following conclusions:

The overall Mental Wellbeing \& Resilience status of a majority the students are normal.

Majority of students are found to be having Medium Mental Wellbeing scores. However a significant percentage of medical students scored Low Mental Wellbeing scores. Surprisingly, a very negligible percentage of students scored High Mental Wellbeing scores. Majority of students are found to be having Normal Resilience scores. However a significant percentage of medical students scored Low Resilience scores. Surprisingly, a very negligible percentage of students scored High Resilience scores. Mental Wellbeing \& Resilience of medical students show significant relation with one another.

The perception of a major percentage of medical students is that, Mental Wellbeing and Resilience will in fact have an effect on their future behavior as a doctor. While a very small percentage believe otherwise. Another perception that a very large percentage of MBBS students hold is that Institutional, Peer Based \& Academic interventions will have a positive effect on their future behavior as doctor.

\section{Acknowledgement}

We are thankful to Allah Almighty, who endowed us with the necessary opportunity and endurance required to undertake and complete this work. We offer our deepest gratitude and sincere thanks our head of department Prof Muzammil Hasan Najmi. For providing a research oriented environment.

We are also extremely thankful to our batch research supervisor Dr.Tahira Amjad for her guidance, dedication and constant supervision, valuable feedback and constant encouragement throughout the duration of the project. Her valuable suggestions were of immense help throughout our project work. Her perceptive criticism kept us working to make this project in a much better way. Working under her supervision was an extremely knowledgeable experience for us.

We are thankful to all those who participated in this research and were very cooperative at the time of data collection which was a tedious job of our research project. 


\section{2nd International Conference on Research in SOCIAL SCIENCES and HUMANITIES}

\section{References}

The World Health Report 2001: Mental Disorders affect one in four people. (2020). $\begin{array}{llll}\text { Retrieved } & 1 & \text { December } & \text { 2020, }\end{array}$ https://www.who.int/whr/2001/media_centre/press_release/en/

Kumar, S. (2020). Burnout and Doctors: Prevalence, Prevention and Intervention. Retrieved 2 December 2020

Zhang, G., Yang, X., Tu, X., Ding, N., \& Lau, J. (2020). Prospective relationships between mobile phone dependence and mental health status among Chinese undergraduate students with college adjustment as a mediator. Retrieved 2 December 2020

Bacchi, S., \& Licinio, J. (2020). Resilience and Psychological Distress in Psychology and Medical Students. Retrieved 2 December 2020

Mental health, suicidal ideation, and experience of bullying among university students in Pakistan - Akhtar Bibi, Simon E Blackwell, Jürgen Margraf, 2019. (2020). Retrieved 2 December 2020

Sarwar, S., Aleem, A., \& Nadeem, M. (2020). Health Related Quality of Life (HRQOL) and its correlation with academic performance of medical students. Retrieved 2 December 2020 , from

Rachelhardeman.com. 2019 [cited 16 October 2019]. Available from: https://www.rachelhardeman.com/writer\#

Mental health. (2020). Retrieved 2 December 2020, from https://www.who.int/westernpacific/health-topics/mental-health

Mental health: strengthening our response. (2020). Retrieved 2 December 2020, from https://www.who.int/news-room/fact-sheets/detail/mental-health-strengthening-ourresponse

Smith, B., Dalen, J., Wiggins, K., Tooley, E., Christopher, P., \& Bernard, J. (2020). The brief resilience scale: Assessing the ability to bounce back. Retrieved 2 December 2020, from

(2020). $\quad$ Retrieved 2 December 2020, from https://uncw.edu/studentaffairs/committees/pdc/documents/the\%20road\%20to\%20resili ence.pdf

(2020). $\quad$ Retrieved 2 December 2020, from https://www.researchgate.net/publication/308798217_Measuring_the_wellbeing_of_health_care_professionals_in_the_Punjab_a_psychometric_evaluation_of_the _Warwick-Edinburgh_Mental_Well-being_Scale_in_a_Pakistani_population

Houpy, J., Lee, W., Woodruff, J., \& Pincavage, A. (2020). Medical student resilience and stressful clinical events during clinical training. Retrieved 2 December 2020, from https://www.semanticscholar.org/paper/Medical-student-resilience-and-stressfulclinical-Houpy-Lee/ba04164632a76beaa914877333357f4d506a1ccb9 